\title{
Timpanoplastía con injerto en mariposa ${ }^{1}$
}

\section{Tympanoplasty with butterfly graft}

\author{
Iván González Y², Cristián Lara $\mathrm{M}^{2}$, Marcela Castillo F³.
}

\begin{abstract}
RESUMEN
La timpanoplastía es una cirugía frecuente para el otorrino. 日 año 1998 el Dr. Eavey presenta la técnica de injerto en mariposa, la que es avalada posteriormente por varios autores y se afirma que es una técnica eficiente, con menor tiempo quirúrgico y mejor confort para el paciente.

Se trata de un estudio prospectivo realizado en el Hospital Dr. Sótero del Río entre los meses de septiembre 2005 a febrero 2006. En el que se realizó esta técnica a 5 pacientes obteniendo como resultado anatómico cierre de la perforación en 4 pacientes y una extrusión del injerto en 1 paciente post supuración ótica. En cuanto al resultado auditivo la mejoría promedio de los PTP aéreos fue de 15,64 dB. Se concluye que esta técnica presenta las ventajas descritas en la literatura, y se deben realizar estudios sucesivos para determinar qué pacientes se benefician mejor de esta técnica comparándolas con las técnicas tradicionales.
\end{abstract}

Palabras Claves: Timpanoplastía, injerto en mariposa.

\section{SUMMARY}

Tympanoplasty is a frequent surgery for the otolaringologist. In 1998 Dr. Eavey presents the technique of butterfly graft, which is later supported by several authors and it is considered an efficient technique, with less surgical time and better comfort for the patient.

It is a prospective study made at Dr. Sótero del Río Hospital between the months of September 2005 and February 2006, where this technique was applied to 5 patients, obtaining as anatomical result the closing of the perforation in 4 patients and an extrusion of the graft in 1 patient post otic supuration. In relation to the auditive result the average improvement of the air PTP's was 15,64 dB. It is concluded that this technique presents the advantages described in the literature, and successive studies must be made to determine which patients obtain a better benefit from this technique as compared to the traditional techniques.

Key words: Tympanoplasty, butterfly graft.

\footnotetext{
${ }^{1}$ Trabajo presentado en el CEPE del Hospital Dr. Sótero del Río, Santiago, Marzo de 2006.

${ }^{2}$ Residente, U.D.A. Otorrinolaringología, Pontificia Universidad Católica de Chile.

${ }^{3}$ Departamento Otorrinolaringología, Hospital Dr. Sótero del Río.
} 


\section{INTRODUCCIÓN}

La timpanoplastía es una cirugía frecuente en el diario quehacer de un servicio de otorrinolaringología. Existen variados abordajes, técnicas e injertos para reparar una perforación timpánica, como son los abordajes retroauricular, endopreauricular, endoaural, técnicas mediales o laterales (undelay, onlay), injertos de fascia, pericondrio o cartílago pericondrio. Ninguno ha demostrado ser mejor y deben adaptarse al tipo de perforación, necesidad de abordaje de la caja, si se trata de una perforación primaria o secundaria, mecanismo de producción perforación, etc.

日 año 1998 el Dr. Eavey ${ }^{1}$, realizó un estudio con 9 pacientes pediátricos en el que describe una nueva técnica destinada a perforaciones pequeñas y medianas no marginales, a la que llamó timpanoplastía con injerto en mariposa con técnica inlay, la que podríamos traducir como transtimpánica.

En el año 2000 el Dr. Lubianca-Neto ${ }^{2}$ publica un estudio con 20 pacientes usando la técnica de injerto en mariposa, agregando algunas modificaciones en adultos.

Desde entonces se han publicado estudios similares y algunos que comparan los resultados con otras técnicas de timpanoplastías ${ }^{3-5}$.

En general todos concluyen que es una técnica eficiente para cerrar perforaciones timpánicas tanto en niños como en adultos, disminuye el tiempo quirúrgico y la morbilidad postoperatoria, con un mejor confort para el paciente. Al compararla con otras técnicas en cuanto a cierre de perforación y mejoría auditiva fueron similares.

\section{OBJETIVOS}

1. Generar experiencia en el uso de esta técnica en un hospital docente.

2. Conocer y difundir esta técnica, la que aparentemente no es usada en el medio nacional.

3. Poder establecer los beneficios preliminares que ofrece.

\section{MATERIALES Y MÉTODOS}

Se trata de un estudio prospectivo realizado en el Servicio de Otorrinolaringología del Hospital Dr. Sótero del Río entre los meses de septiembre 2005 a febrero 2006.

Se seleccionó un grupo de 5 pacientes que presentaban perforaciones timpánicas descritas como adecuadas para esta técnica, es decir, perforación timpánica de pequeña a mediana, con buen reborde timpánico y en los cuales no se plantea revisión de cadena, o exploración de la caja timpática.

Se les realizó, previo consentimiento informado, timpanoplastía con injerto en mariposa a todos los pacientes por el mismo cirujano (residente de tercer año).

\section{Técnica quirúrgica}

Se realiza bajo anestesia general en 4 pacientes y local en 1 paciente.

- $\quad$ 日 paciente es infiltrado con lidocaína al $2 \%$ y epinefrina 1:100.000 sobre la piel tragal y adicionalmente en la pared posterosuperior del C.A.E para los pacientes operados con anestesia local más sedación.

- Se visualiza con microscopio por vía endoaural la perforación y la mucosa de la caja con el fin de descartar contraindicaciones de esta técni$\mathrm{ca}$

- Con un ganchito o guadaña se realizan cortes alrededor del margen de la perforación en la forma de un prepicado. Figura 1.

- Luego con una pinza de Hartman y ayudado por la aspiración se procede a retirar todo el epitelio del borde de la perforación.

- A continuación ayudados de un bisturí de conducto $\mathrm{N}^{\circ} 1$ se mide el diámetro de la perforación.

- Se procede a tomar el injerto de cartílago tragal de la forma habitual.

- Se talla el injerto de cartílago con un diámetro de aproximadamente $2 \mathrm{~mm}$, mayor al tamaño de la perforación, ayudados con un otoscopio.

- A continuación ayudados de una pinza Adson y un bisturí $N^{\circ} 11$ se realiza una incisión en todo 


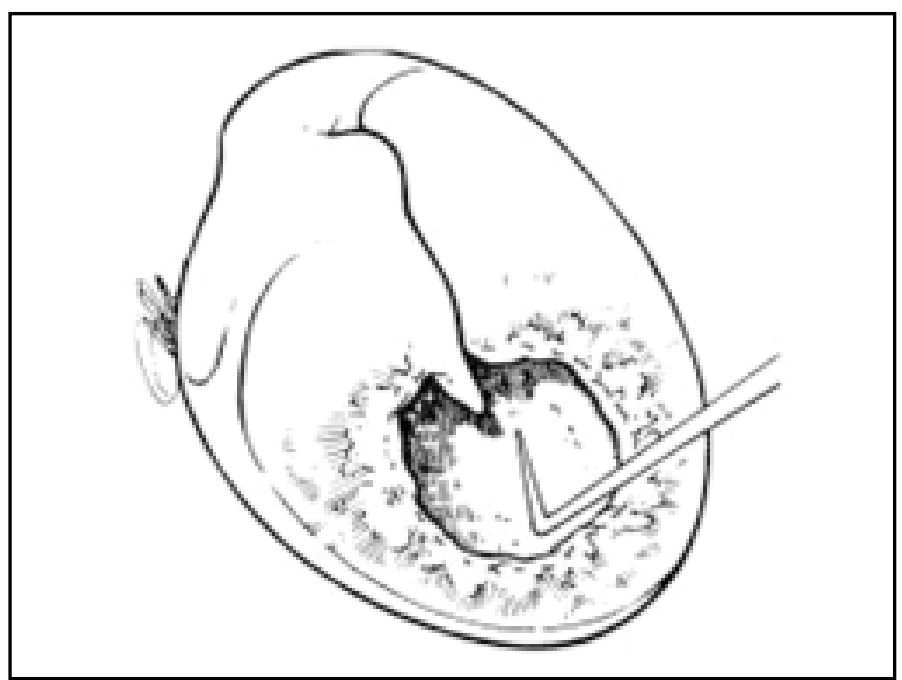

Figura 1.

el borde circunferencial con lo que el cartílago se abre remedando la forma de una mariposa, o para nosotros (a modo chileno) de un alfajor. Figura 2.

- Adicionalmente se reseca una cuña de cartílago en una de sus caras para facilitar su inserción.

- Luego con un fórceps y en una manera similar a la instalación de un tubo de ventilación se pone el injerto transtimpánico, luego ayudados de un ganchito se revisa el borde del injerto para comprobar un buen ajuste. Figura 3.

- Fnalmente aplicamos gelita sobre el injerto y se pone un pequeño parche sobre el pabellón que cubre el conducto.

\section{RESULTADOS}

Se realizó esta técnica en 5 pacientes, la distribución por sexo se ve en la Figura 4.

Con un promedio de edad de 27,8 años (10 a 49 años).

日 tiempo operatorio fue en promedio 45 minutos (30-60).
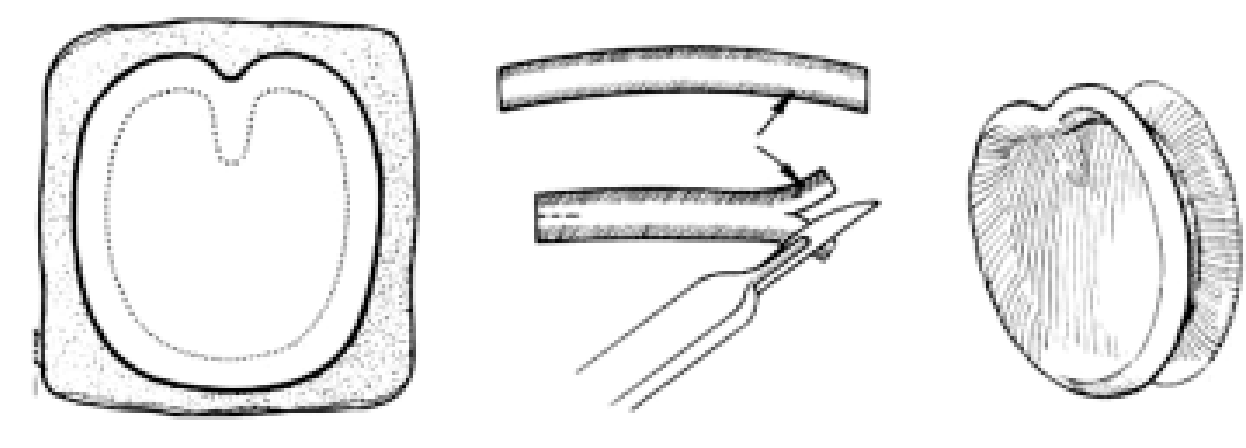

Figura 2. 


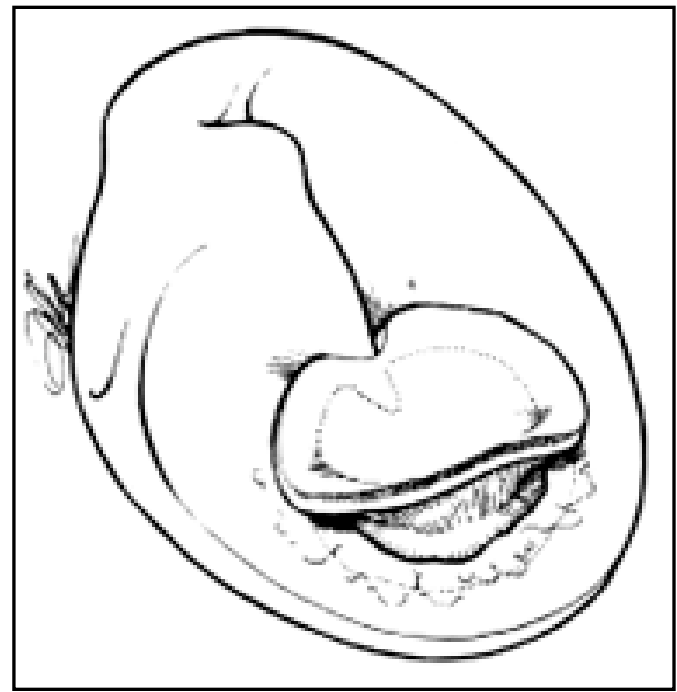

Figura 3.

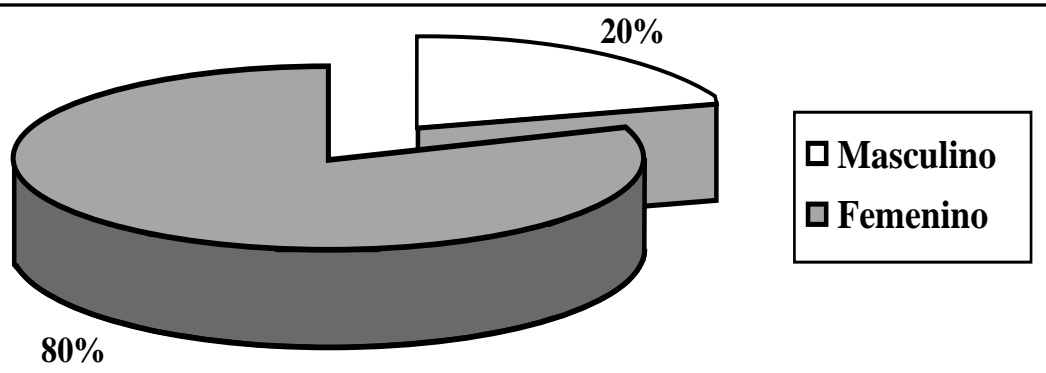

Figura 4. Distribución por sexo.

日 resultado anatómico al último control en febrero de 2006, 4 pacientes presentaron tímpano cerrado con el injerto integrado y en un paciente se observó que luego de una otitis externa con otorrea abundante, atribuible a piscina, una extrusión del injerto hacia el CAE

La audiometría pre y post se muestra en la Tabla 1.

La audiometría postoperatoria se tomó a los tres meses de evolución.

\section{CONCLUSIONES}

- Larealización de estetrabajo nos permitió familiarizarnos con la técnica, laque rápidamente logramos dominar. Logrando realizarla de manera expedita

- Oreemos que ésta es una técnica fácil, rápida, no requiere de incisiones, salvo la incisión tragal, por lo que el resultado cosmético es excelente, y más confortable para el paciente.

- No requiere de gelita u otro material dentro de la caja timpánica por lo que la sensación auditiva, una vez retirado el parche, es muy agradable para el paciente.

- En términos de ahorro de tiempo anestésico, y la posibilidad de realizarla en forma ambulatoria, resulta una técnica atractiva económicamente para un servicio de otorrino.

- $\quad$ r resultado obtenido en cuanto al cierre de la perforación y mejoría auditiva aparentemente es similar al obtenido con otras técnicas. 


\section{Tabla 1.}

\begin{tabular}{|lrrrr|}
\hline Ódo Operado & $\begin{array}{r}\text { Audiometría } \\
\text { aérea }\end{array}$ & $\begin{array}{r}\text { Pre Op } \\
\text { ósea }\end{array}$ & $\begin{array}{r}\text { Audiometría } \\
\text { aérea }\end{array}$ & $\begin{array}{r}\text { Post Op. } \\
\text { ósea }\end{array}$ \\
\hline I & 33,3 & 10,6 & 20 & 10,6 \\
I & 50 & 15 & 33 & 15 \\
D & 28,3 & 15 & 15 & 15 \\
D & 1,6 & 5 & 1,6 \\
D & 13,6 & 8,3 & 45,3 & 8,3 \\
Promedio & 45,3 & 10,1 & 23,66 & 10,1 \\
\hline
\end{tabular}

Con una mejoría promedio de los PTP de 15,64 dB.

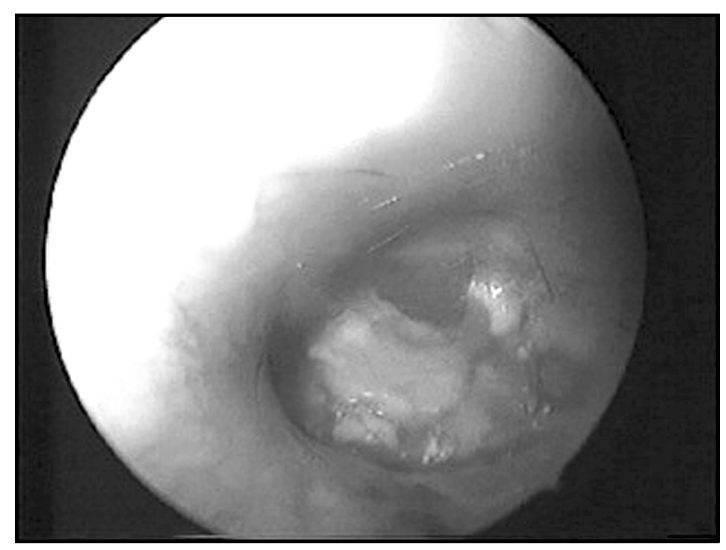

Figura 5. Control al tercer mes postoperatorio, paciente masculino de 15 años de edad.

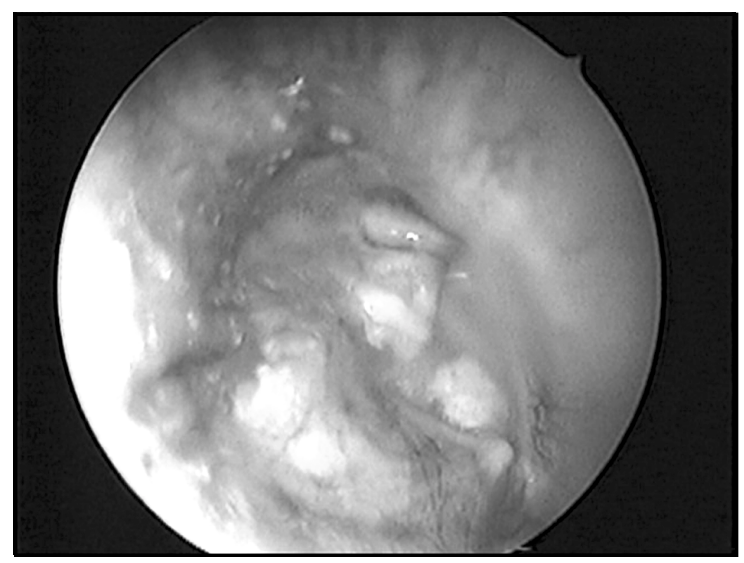

Figura 6. Control al sexto mes postoperatorio, paciente femenino de 46 años de edad. 


\section{DISCUSIÓN}

Estamos optimistas con los resultados obtenidos en el presente estudio, ya que son similares a lo descrito en la literatura.

Sabemos que es una serie muy pequeña, por lo tanto los resultados son difíciles de interpretar. A futuro quisiéramos poder identificar quiénes y qué tipo de perforaciones son las que se beneficiarían de esta nueva técnica

Para poder obtener resultados más objetivos y comparables con las otras técnicas de timpanoplastía se debe realizar estudios con mayor número de pacientes y que comparen las distintas técnicas.

Creemos que ésta es una técnica más en el arsenal del Otorrinolaringólogo para enfrentar la otitis media crónica.

Fnalmente cabe comentar que la técnica original descrita por el Dr. Eavy utiliza injerto de cartílago y pericondrio, reforzado con piel del antebrazo, posteriormente el Dr. Lubianca-Neto la modifica usando sólo el injerto de cartílago-pericondrio y nosotros utilizamos sólo cartílago.

\section{BIBLIOGRAFÍA}

1. EAVEY, ROAND D, MD. Inlay Tympanoplasty: Cartilage Butterfly Technique. Laryngoscope 1998; 108(5): 657-61.

2. LUBIANCA-NETOJF. Inlay Butterfly Cartilage Tympanoplasty (Eavey technique) modified for adults. Otolaryngol Head Neck Surg 2000; 123 (4): 492-4.

3. MAURI M, LUBIANCA-NETO JF, FuaHS SC. Evaluation of Inlay Butterfly Cartilage Tympanoplasty: a randomized clinical trial. Laryngoscope2001; 111(8): 1479-85.

4. TS ANAND, G\#TA KaTHURIA, SAND巴 KUMAR, VIKRAMWADHWA AND TAPASWINI PRADHAN. Butterfly Inlay Tympanoplasty: a study in Indian scenario. Indian Journal of Oolaryngology and Head and Neck Surgery 2002; 54(1): 11-13.

5. COUloigneR VinCent, BaCUlard Florence, E BaKkouri, Wissame, Viala PaUl, francols Martine, Nancy P, Van Den Abbeele T. Inlay Butterfly Cartilage Tympanoplasty in children. Otology and Neurotology 2005; 26(2): 247-51.

Dirección: Dr. Iván González

Teléfono: 09-7237735

Email: ivangy@gmail.com 